\title{
Suppression of glutamate synthase genes significantly affects carbon and nitrogen metabolism in rice (Oryza sativa L.)
}

\author{
LU YongEn, LUO Feng, YANG Meng, LI XiangHua \& LIAN XingMing* \\ National Key Laboratory of Crop Genetic Improvement, National Center of Plant Gene Research (Wuhan), \\ Huazhong Agricultural University, Wuhan 430070, China
}

Received March 24, 2011; accepted June 16, 2011

\begin{abstract}
Rice (Oryza sativa) glutamate synthase (GOGAT, EC 1.4.1.14) enzymes have been proposed to have great potential for improving nitrogen use efficiency, but their functions in vivo and their effects on carbon and nitrogen metabolism have not been systematically explored. In this research, we analyzed transcriptional profiles of rice GOGAT genes using a genome-wide microarray database, and investigated the effects of suppression of glutamate synthase genes on carbon and nitrogen metabolism using GOGAT co-suppressed rice plants. Transcriptional profiles showed that rice GOGAT genes were expressed differently in various tissues and organs, which suggested that they have different roles in vivo. Compared with the wild-type, tiller number, total shoot dry weight, and yield of GOGAT co-suppressed plants were significantly decreased. Physiological and biochemical studies showed that the contents of nitrate, several kinds of free amino acids, chlorophyll, sugars, sugar phosphates, and pyridine nucleotides were significantly decreased in leaves of GOGAT co-suppressed plants, but the contents of free ammonium, 2-oxoglutarate, and isocitrate in leaves were increased. We conclude that GOGATs play essential roles in carbon and nitrogen metabolism, and that they are indispensable for efficient nitrogen assimilation in rice.
\end{abstract}

\begin{abstract}
glutamate synthase, Oryza sativa, co-suppression, carbon and nitrogen metabolism
\end{abstract}
Citation: $\quad$ Lu Y E, Luo F, Yang M, et al. Suppression of glutamate synthase genes significantly affects carbon and nitrogen metabolism in rice (Oryza sativa L.). Sci China Life Sci, 2011, 54: 651-663, doi: 10.1007/s11427-011-4191-9

Nitrogen is one of the major limiting nutrients for the growth and development of rice (Oryza sativa). Maintaining or increasing world-wide rice production is strongly dependent on the use of nitrogen fertilizers, which creates economic and environmental pressure for research on improving nitrogen use efficiency [1].

Glutamine 2-oxoglutarate aminotransferase (glutamate synthase, GOGAT) and glutamine synthetase (GS) are the key enzymes in primary assimilation and re-assimilation of nitrogen in plants. GS catalyzes the condensation reaction of one molecule of ammonium and one molecule of glutamate (Glu) to form one molecule of glutamine (Gln). GOGAT catalyzes the reaction of one molecule of Gln and one

*Corresponding author (email: xmlian@mail.hzau.edu.cn) molecule of 2-oxoglutarate (2-OG) to form two molecules of Glu. These two reactions coupled together are known as the GS/GOGAT cycle, through which inorganic nitrogen is transformed into organic nitrogen. The two kinds of GOGAT in higher plants, NADH-GOGAT (EC 1.4.1.14) and Fd-GOGAT (EC 1.4.7.1), use NADH and ferredoxin $(\mathrm{Fd})$, respectively, as the electron carrier. NADH-GOGAT is primarily located in plastids of non-photosynthetic tissues such as roots, and Fd-GOGAT is located predominantly in leaf chloroplasts [2]. In root nodules of legumes, NADHGOGAT assimilates the nitrogen fixed by Rhizobium; in non-legumes, it functions in primary nitrogen assimilation or re-assimilation of ammonium released during amino acid catabolism. The major physiological role of Fd-GOGAT was suggested to be re-assimilation of the ammonium liber- 
ated in photorespiration [2]. cDNA clones of NADHGOGAT have been isolated from several plants such as alfalfa (Medicago sativa), Arabidopsis thaliana, rice [3], and soybean (Glycine max) [4]. Fd-GOGAT cDNAs have been cloned from maize (Zea mays), tobacco (Nicotiana tabacum), barley (Hordeum vulgare), spinach (Spinacia oleracea), Scotch pine (Pinus sylvestris), and Arabidopsis [2].

Two expressed Fd-GOGAT genes (AtGLU1 and AtGLU2) and one NADH-GOGAT gene (AtGLT1) were reported in Arabidopsis [5,6]. Analyses of Arabidopsis gls mutants revealed that the major role of AtGLU1 is to re-assimilate the ammonium released in photorespiration. AtGLU1 may also have a role in primary nitrogen assimilation in leaves, whereas $A t G L U 2$ has a major role in primary nitrogen assimilation in roots [5]. AtGLT1 mRNA is expressed at higher levels in roots than in leaves, which suggested its functions in assimilating non-photorespiratory ammonium and providing Glu for plant development [6]. Blast analyses in NCBI (http://www.ncbi.nlm.nih.gov/) and KOME (http:// cdna01.dna.affrc.go.jp/cDNA/) also indicated that rice has two Fd-GOGAT genes: OsFd-GOGATI is expressed mainly in green leaves [7], but also in roots [8,9]. As reported by Suzuki et al. [7], OsFd-GOGAT2 probably encodes a different Fd-GOGAT protein in roots, and it has different immunological properties from Fd-GOGAT1 in leaves. The in vivo function of $O s F d-G O G A T 2$ remains unknown. Two expressed NADH-GOGAT genes are also present in the rice genome. OsNADH-GOGATI mRNA is expressed mainly in developing tissues, such as premature leaf blades, spikelets at the early stage of ripening, and roots [10,11], and rice NADH-GOGAT activity is also high in these tissues [12-14]. In roots, the transcript of OsNADH-GOGAT1 and the protein it encodes can be induced by supplying ammonium $[8,9,15,16]$. OsNADH-GOGAT2 is expressed only in the leaf and leaf sheath at the transcript level [11].

The GS/GOGAT cycle sits at the interface of carbon and nitrogen metabolism. Over-expression of NADH-GOGAT and GS has been proposed as a strategy to improve nitrogen use efficiency [1]. For this purpose, a great deal of research has focused on GS over-expression, with encouraging results reported [17]. Because GOGAT genes are too large to manipulate easily, only a few research results have been reported $[18,19]$. However, these studies did not systematically analyze how GOGAT genes affect carbon and nitrogen metabolism in vivo.

To further explore the functions of the GOGAT genes in rice, we first analyzed the transcriptional profiles of four rice GOGAT genes in 25 different tissues or organs using a genome-wide microarray database. Then we used GOGAT co-suppressed transgenic rice plants, in which both $O s$ $N A D H-G O G A T$ and $O s F d-G O G A T$ were significantly suppressed, to systematically investigate the effects of suppression of GOGAT genes on carbon and nitrogen metabolism. Transcriptional profiles showed that members of the rice GOGAT gene family were expressed differently in various tissues and organs, indicating that they have different functions in vivo. Suppression of GOGAT genes resulted in significant decreases in tiller number, total shoot dry weight, and yield per plant. The contents of nitrate, several kinds of free amino acids, chlorophyll, sugar, sugar phosphates, pyridine nucleotides in leaves of co-suppressed plants were also significantly decreased, but the contents of free ammonium, 2-OG, and isocitrate in leaves were increased. We conclude that glutamate synthases play essential roles in carbon and nitrogen metabolism, and that they are indispensable for the efficient nitrogen assimilation process in rice. The relationships between GOGAT functions and these effects were assessed.

\section{Materials and methods}

\subsection{Transcriptional profiling and phylogenetic analysis of $G O G A T$ genes in rice}

The transcription profile data of the rice GOGAT-family genes in 25 tissues or organs of three rice cultivars (Minghui 63, Zhenshan 97, and Shanyou 63) were retrieved from the CREP database (http://crep.ncpgr.cn). For the GOGAT protein sequences, a phylogenetic tree was generated using ClustalX (Version 1.83) with the neighbor-joining method and bootstrap analysis with 1000 replicates. The tree was analyzed and displayed using MEGA software (Version 4).

\subsection{Plasmid construction and rice transformation}

A genomic DNA fragment of OsNADH-GOGAT1 was amplified from the Nipponbare BAC clone a0079a11 using primers Glt1F and Glt1R. Both primers contained an Nhe I restriction site (Table 1). This fragment was then cloned into the multiple cloning sites (MCS) of the pCAMBIA1301S vector between the CaMV 35S promoter and the nopaline synthase terminator (Nos polyA) in the sense orientation. The vector pCAMBIA1301S was modified by us based on pCAMBIA1301 (obtained from the Centre for the Application of Molecular Biology to International Agriculture, CAMBIA, Australia). Standard molecular biology techniques were used for DNA manipulation. The chimeric fragment was introduced into the japonica rice cultivar Zhonghua 11 by an Agrobacterium-mediated transformation method [20]. Polymerase chain reactions were carried out with specific primers to identify positive transgenic plants; the forward primer $1301 \mathrm{sF}$ was designed according to the pCAMBIA 1301s vector sequence, and the reverse primer was designed according to the sequence of the $O s$ NADH-GOGAT1 fragment (Table 1).

\subsection{Plant material, growth conditions, and sampling}

For hydroponic culture, seeds of transgenic plants and 
Table 1 Primers used in experiments

\begin{tabular}{cclc}
\hline Gene or vector name & Primer name & \multicolumn{1}{c}{ Primer sequence $\left(5^{\prime}-3^{\prime}\right)$} & Function \\
\hline OsNADH-GOGAT1 & Glt1F & GGTGGCTAGCGTACGGTCTAATGCTCGTCGTTTGATGC & Gene amplification \\
& Glt1R & CTCTGCTAGCGTGGTGACATTTACTCCTGATGGCCAAG & Gene amplification \\
& Positive NA1 & ACTGCAGACGTGGACAATCACT & Transformant identification \\
& Nor-Glt1F & GGCTGCTCAGTACTGTGTTGAA & Northern blot probe \\
& Nor-Glt1R & GTTCCTTTGCAAGCTTAGCAG & Northern blot probe \\
OsFd-GOGAT1 & Glu1F & TGGTTGAGGGCACTGGAGATCATT & Real-time RT-PCR \\
& Glu1R & AATATAGGCAAGGCCACCCGTCAT & Real-time RT-PCR \\
OsNADH-GOGAT2 & Re-Glt2F & CCTGTCGAAGGATGATGAAGGTGAAACC & Real-time RT-PCR \\
& Re-Glt2R & TGCATGGCCCTACTATCTTCGCATCA & Real-time RT-PCR \\
1301s vector & 1301sF & CACACAGGAAACAGCTATGACC & Transformant identification \\
GUS & GUSF & ACGACTCGTCCGTCCTGTAGAA & Southern blot probe \\
& GUSR & CGGTTCGTTGGCAATACTCC & Southern blot probe \\
\hline
\end{tabular}

wild-type Zhonghua 11 plants were germinated and sown in sand. Young seedlings at the 2-leaf stage were transferred to Yoshida culture solution [21], and the solution was refreshed once a week. For field experiments, transgenic lines, wild-type, and segregated negative lines were planted in the field following a randomized complete block design with three replications. Each plot contained 30 plants of each genotype. Agronomic trait data were collected after maturity. Normal agricultural practices were followed. For real-time RT-PCR tests and enzyme activity assays, leaves and roots were sampled from hydroponic plants at the 5-6 leaf stage. For metabolite tests, leaves were sampled from $10: 00$ to $11: 30$ in the morning and from $22: 00$ to $0: 00$ at night $38 \mathrm{~d}$ after transplanting in the field.

\subsection{Southern blot and Northern blot analysis}

Genomic DNA was extracted from $\mathrm{T}_{0}$ transgenic plants using the CTAB method, and $5 \mu \mathrm{g}$ genomic DNA was digested with Hind III. The DNA was then transferred to Hybond $\mathrm{N}^{+}$nylon membranes (Amersham, Buchinghamshire, UK) for Southern blot analysis. Total RNA was extracted from the leaves with TriZol reagent (Invitrogen, Gaithersburg, MD, USA) according to the manufacturer's instructions; $15 \mu \mathrm{g}$ total RNA was then used for Northern blot analysis. Hybridizations were performed as described by Sambrook and Russell [22], with a ${ }^{32} \mathrm{P}$-labeled specific fragment of OSNADH-GOGATI coding sequence as the probe for Northern blot analysis, and a ${ }^{32} \mathrm{P}$-labeled GUS gene fragment as the probe for Southern blot analysis (primers in Table 1). The results were detected by autoradiography.

\subsection{Enzyme assays}

Leaf and root samples were taken from hydroponic plants and kept on ice. A 0.2-g sample was homogenized on ice with a mortar and pestle, with a small amount of quartz sand and $1.5 \mathrm{~mL}$ extraction buffer $\left(50 \mathrm{mmol} \mathrm{L}{ }^{-1}\right.$ Tris- $\mathrm{HCl} \mathrm{pH} 7.5$, $10 \mathrm{mmol} \mathrm{L}^{-1} \mathrm{MgCl}_{2}, 1 \mathrm{mmol} \mathrm{L}{ }^{-1}$ EDTA, $1 \mathrm{mmol} \mathrm{L}{ }^{-1}$ EGTA,
$1 \mathrm{mmol} \mathrm{L}{ }^{-1}$ benzamidine, $1 \mathrm{mmol} \mathrm{L}{ }^{-1} \varepsilon$-aminocaproic acid, $1 \mathrm{mmol} \mathrm{L}{ }^{-1}$ PMSF (added just before extraction), and 10 $\mu \mathrm{mol} \mathrm{L} \mathrm{L}^{-1}$ leupeptin). The homogenate was collected into a 2.0-mL microcentrifuge tube, and after centrifugation at $16000 \times g$ for $16 \mathrm{~min}$ at $4^{\circ} \mathrm{C}$, the supernatant was used for enzyme assays. NADH-GOGAT activity was assayed as described by Hecht et al. [23]. Ala aminotransferase (AlaAT), Asp aminotransferase (AspAT), fructose-1,6-bisphosphatase (FBPase), glucokinase (GlcK), fructokinase (FruK), NADPdependent glucose-6-phosphate dehydrogenase (G6PDH), NADP-dependent isocitrate dehydrogenase (NADP-ICDH), shikimate dehydrogenase (ShikDH), and phosphoenolpyruvate carboxylase (PEPC) were assayed in conditions as described in Gibon et al. [24] except that the reactions were monitored directly at $340 \mathrm{~nm}$. Fd-GOGAT, nitrate reductase (NR) and GS were assayed as described by Gibon et al. [24] and Migge et al. [25], respectively. We used the method of Scheible et al. [26] to assay nitrite reductase (NiR) and that of Turano et al. [27] to assay glutamate dehydrogenase (GDH). Malate dehydrogenase (NADP-MDH) was assayed as described by Jenner et al. [28] and pyruvate kinase (PK) as described by Borsani et al. [29].

\subsection{Metabolite analysis}

Soluble sugars, sugar phosphates, and organic acids in leaf samples were extracted as described by Schneidereit et al. [30]. Isocitrate, 2-OG, pyruvate, and oxaloacetate (OAA) were determined by quantifying $\mathrm{NAD}(\mathrm{H})$ or NADP $(H)$ by the PES-MTT method after appropriate enzymatic reactions [31]. Malate was measured as described by Novitskaya et al. [32]. Sugar phosphates were determined according to Gibon et al. [31]. Glucose, fructose, and sucrose concentrations were determined using the Megazyme K-SUFRG assay kit (Megazyme International Ireland Ltd., Bray Business Park, Bray, Co., Wicklow, Ireland). Pyridine nucleotides were extracted and determined as described by Gibon and Larher [33]. Leaf nitrate content was determined according to the method of Dutilleul et al. [34]. Ammonium was determined using the Megazyme K-AMIAR 
assay kit. Leaf free amino acids were extracted from ground leaf powder into $80 \%$ ethanol and quantified using a Hitachi amino acid analyzer (L-8800, Hitachi Instruments Engineering, Tokyo, Japan) according to the manufacturer's instructions. Leaf starch was determined as described by Wang et al. [35]. Total leaf soluble sugar was measured using the anthrone- $\mathrm{H}_{2} \mathrm{SO}_{4}$ method [36] and total leaf soluble protein using the Bradford method [37] with bovine serum albumin as the standard.

\subsection{Relative real-time RT-PCR analysis}

The RNA samples were treated with RNase-free DNase I (Invitrogen) to remove any residual genomic DNA in the preparation. First-strand DNA synthesis was carried out using $3 \mu \mathrm{g}$ total RNA as the template, with SuperScript II reverse transcriptase (Invitrogen) and oligo dT (Promega, Madison, WI, USA). The product was diluted to a final volume of $80 \mu \mathrm{L}$. Primers for real-time RT-PCR were designed using Primer Express 2.0 software (Table 1). Real-time RT-PCR was performed in an optical 96-well plate with an ABI PRISM 7500 real-time PCR system (Applied Biosystems, Foster City, CA, USA). Each reaction contained 12.5 $\mu \mathrm{L}$ SYBR Premix Ex Taq (Takara, Kyoto, Japan), $0.5 \mu \mathrm{L}$ ROX Reference Dye II (Takara), $1.0 \mu \mathrm{L}$ cDNA sample, and $1.0 \mu \mathrm{L} 10 \mu \mathrm{mol} \mathrm{L}{ }^{-1}$ gene-specific primers in a final volume of $25 \mu \mathrm{L}$. The thermal cycle was as follows: $95^{\circ} \mathrm{C}$ for $30 \mathrm{~s} ; 40$ cycles of $95^{\circ} \mathrm{C}$ for $5 \mathrm{~s}$, and $60^{\circ} \mathrm{C}$ for $34 \mathrm{~s}$. Data were collected during the extension step $\left(60^{\circ} \mathrm{C}\right.$ for $\left.34 \mathrm{~s}\right)$. The rice gene Actin was used as an internal control with the primers ActinF and ActinR (Table 1). The $2^{-\Delta \Delta C_{\mathrm{T}}}$ method was used for the analysis of real-time RT-PCR data [38].

\subsection{Measurement of chlorophyll content and $\boldsymbol{F}_{v} / \boldsymbol{F}_{m}$}

Chlorophyll content was determined by extracting pigments into $80 \%$ acetone and then measuring the absorbance of the solution at 663 and $646 \mathrm{~nm}$. Chlorophyll fluorescence data
$\left(F_{v} / F_{m}\right)$ was obtained as described by Werner et al. [39] with a FMS2 chlorophyll fluorometer (Hansatech Instruments Ltd., Norfolk, UK).

\subsection{Statistics}

The statistical significance of differences between data from wild-type and transformed plants was determined by Student's $t$-test using SPSS 12.0 software.

\section{Results}

\subsection{GOGAT genes and their transcriptional profiles in rice}

After searching in the NCBI, KOME, and TIGR databases, we identified two Fd-GOGAT genes (XM_479407 for OsFd-GOGAT1 and AK110476 for OsFd-GOGAT2) and two NADH-GOGAT genes (AB008845 for OsNADHGOGAT1 and LOC_Os05g48200.1 for OsNADH-GOGAT2) in rice. Alignment of their deduced amino acid sequences showed that rice NADH-GOGAT2 shared $80 \%$ identity with rice NADH-GOGAT1. Rice NADH-GOGAT1 and NADH-GOGAT2 shared $43 \%$ and $42 \%$ identity with rice Fd-GOGAT1, respectively (Figure 1).

Data on the transcription profiles of these genes in 25 tissues or organs of Minghui 63, Shanyou 63, and Zhenshan 97 were retrieved from the CREP database (http://crep. ncpgr.cn). Most of the results showed that transcription profiles of GOGAT genes were similar to those described previously [12-14], but there were some differences. Besides roots, OsNADH-GOGAT1 was expressed at relatively high levels in stamens $1 \mathrm{~d}$ before flowering but weakly in the endosperm after pollination (Figure 2A), indicating that it may be involved in providing nitrogen or amino acids for stamen development. OsNADH-GOGAT2 was mainly expressed in leaves and leaf sheaths; it was also highly expressed in endosperm 14 and $21 \mathrm{~d}$ after pollination but was expressed at a low level in endosperm $7 \mathrm{~d}$ after pollination

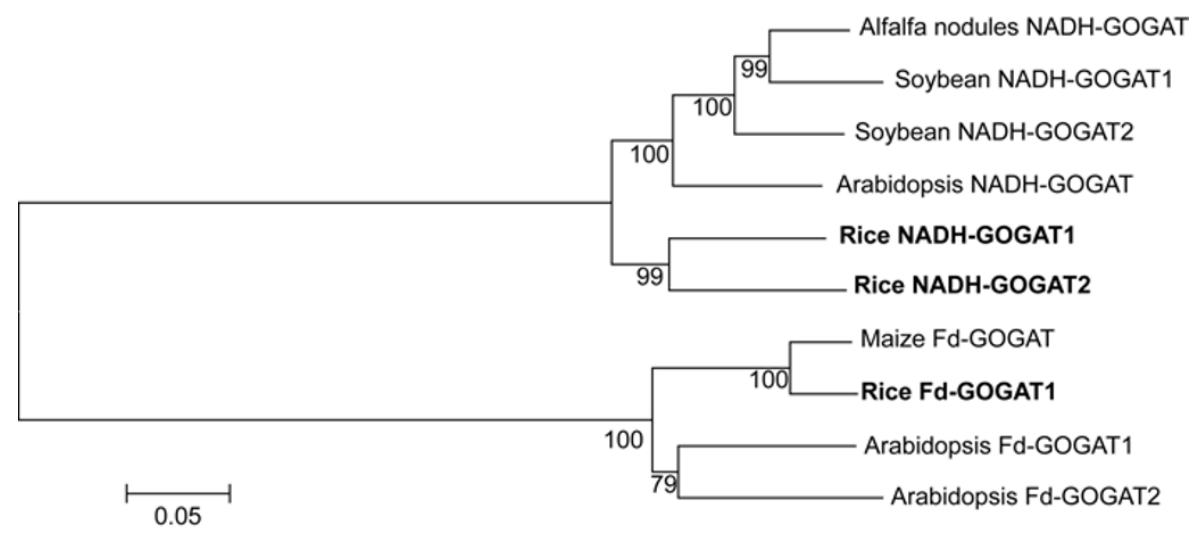

Figure 1 Phylogenetic analysis of GOGAT proteins. The phylogenetic tree was generated using ClustalX by the neighbor-joining method. Bootstrap values from 1000 replicates are indicated at each node. Organelle targeting sequences were removed from the aligned sequences. 

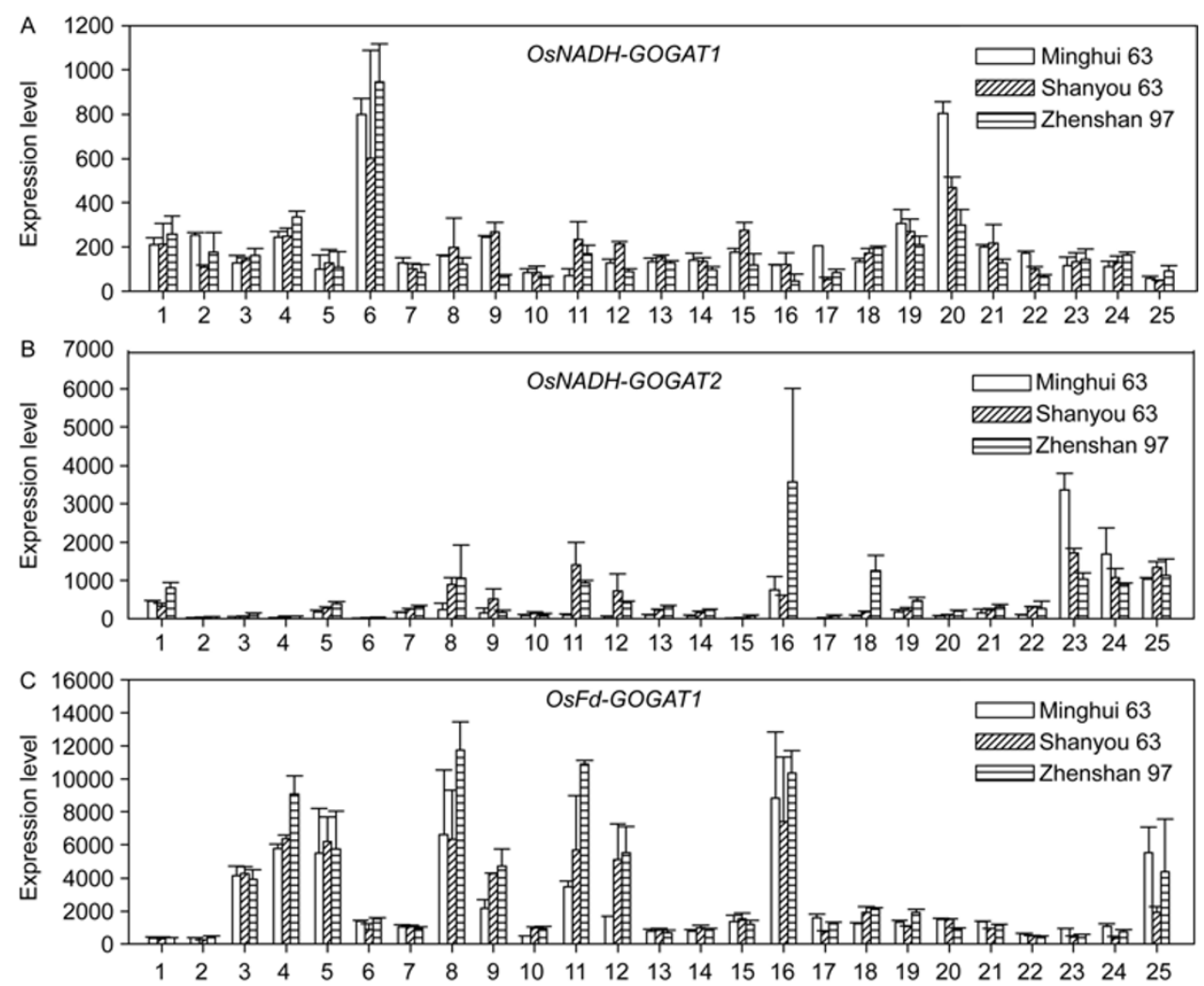

Figure 2 Expression patterns of rice GOGAT genes in Minghui 63, Shanyou 63, and Zhenshan 97. The $X$-axis represents various tissues and the $Y$-axis represents raw expression values obtained from microarray. Numbers on $X$-axis correspond to the following tissues: 1 , seed: germination ( $72 \mathrm{~h}$ after imbibition); 2, calli: $15 \mathrm{~d}$ after induction; 3, seedling 1: $3 \mathrm{~d}$ after sowing; 4, seedling 2: trefoil stage; 5 , shoot 1: seedling with 2 tillers; 6 , root 1: seedling with 2 tillers; 7 , panicle 1: secondary branch primordium differentiation stage; 8 , leaf 1 : secondary branch primordium differentiation stage; 9 , sheath 1 : secondary branch primordium differentiation stage; 10 , panicle $2: 4-5 \mathrm{~cm}$ young panicle; 11 , leaf 2: 4-5 cm young panicle; 12, sheath 2: 4-5 cm young panicle; 13, panicle 3: pistil/stamen primordium differentiation stage; 14 , panicle 4: pollen-mother cell formation stage; 15 , stem 1: $5 \mathrm{~d}$ before heading; 16 , flag leaf 3: 5 $\mathrm{d}$ before heading; 17 , stem 2: heading stage; 18, panicle 5: heading stage; 19 , glume: $1 \mathrm{~d}$ before flowering; 20 , stamen: $1 \mathrm{~d}$ before flowering; 21 , spikelet: $3 \mathrm{~d}$ after pollination; 22, endosperm 1: $7 \mathrm{~d}$ after pollination; 23, endosperm 2: $14 \mathrm{~d}$ after pollination; 24, endosperm 3: $21 \mathrm{~d}$ after pollination; 25 , flag leaf 4: $14 \mathrm{~d}$ after heading.

(Figure 2B). This pattern of expression has not been reported previously. Rice NADH-GOGAT2 may also be involved in providing nitrogen or amino acids for seeds. OsFd-GOGAT1 was expressed in most of the tissues, but mainly in the leaf and leaf sheath (Figure 2C), suggesting that it may re-assimilate ammonium released from photorespiration. OsFd-GOGAT2 transcripts were expressed at low levels in every tissue tested, but was not expressed at higher levels in any particular tissue type (data not shown).

\subsection{Molecular identification of transformants}

More than 50 putative $\mathrm{T}_{0}$ transgenic plants were generated through Agrobacterium-mediated transformation. PCR analysis showed that $>85 \%$ of the plants were positive transformants, and approximately $1 / 3$ of these PCR-checked positive transformants showed phenotypic changes. Northern blot analysis proved that all the positive transformants with phenotypic changes accumulated degraded mRNA of OsNADH-GOGAT, which caused co-suppression (Figure $3 \mathrm{~A})$, whereas no accumulation of degraded mRNA was found in plants without phenotypic changes. The probe was specifically designed for OSNADH-GOGAT1, and no signals were detected in wild-type plants, possibly because of its low expression level in the leaves (Figure 2A). The co-suppressed lines were NA1-4, NA1-5, NA1-7, NA1-8, NA1-11, NA1-17, NA1-20, NA1-40, NA1-50, NA1-68, NA1-72, NA1-75, NA1-81, and NA1-95. Southern blot analysis showed that most of these contained a single copy of the transgene (Figure 3B).

Transcription levels of OsNADH-GOGAT2 and OsFdGOGAT1 were also checked by real-time RT-PCR. Both OsNADH-GOGAT2 and OsFd-GOGAT1 transcripts were significantly suppressed, as compared to their respective levels in the wild-type (Figure 4). Suppression of $O S N A D H$ GOGAT2 was more pronounced, showing only $1.0 \%-6.7 \%$ of its transcription level in the wild-type.

\subsection{Co-suppression caused apparent phenotype and decreased NADH-GOGAT activity}

GOGAT co-suppressed plants showed significantly decreased 

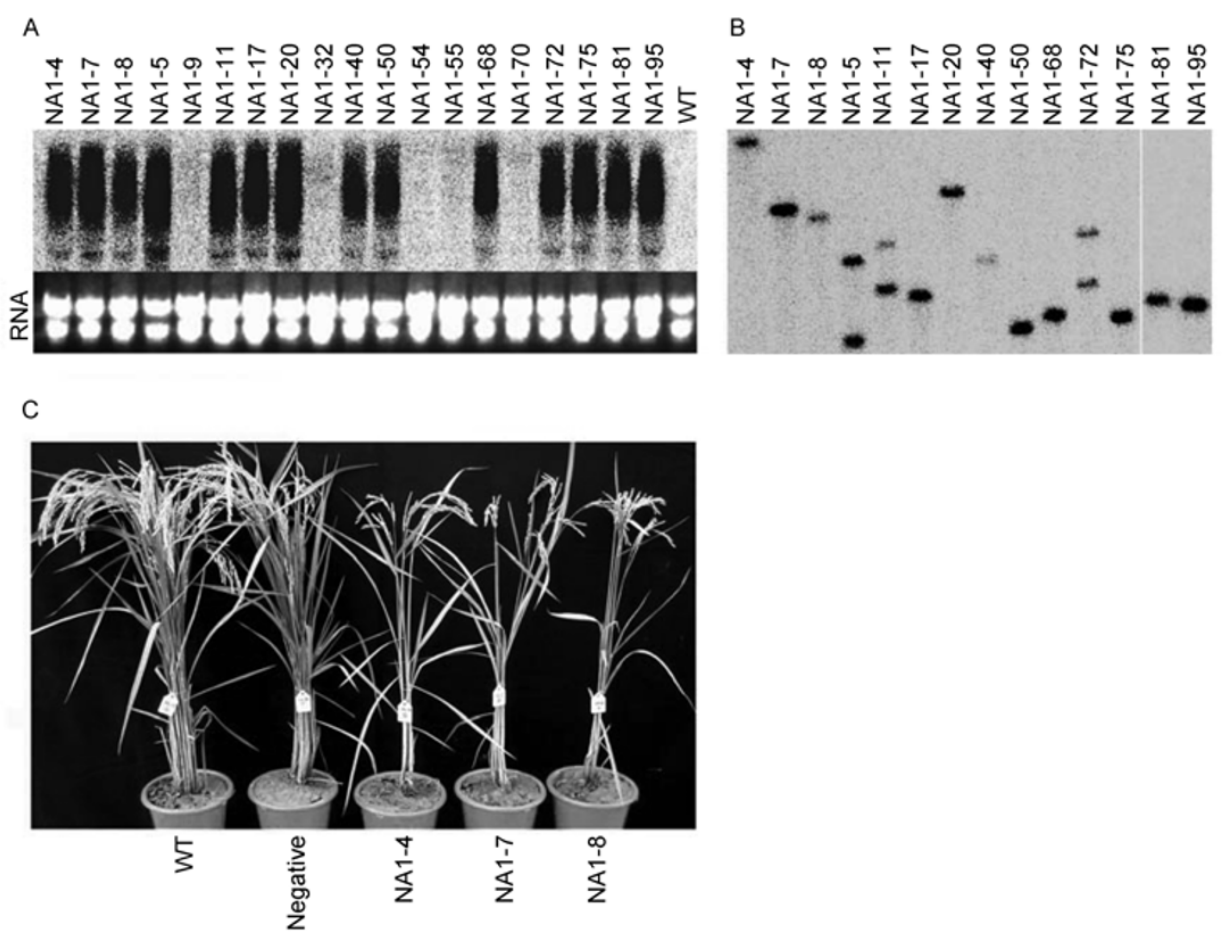

Figure 3 Characterization and transcription of $O S N A D H-G O G A T$ in co-suppressed lines and the wild-type. A, Northern blot analysis of $O s-$ $N A D H-G O G A T 1$ transcript levels in transgenic plants. Total RNA extracted from leaves was probed with a fragment of the coding sequence of the transgene. B, Southern blot analysis with GUS gene probe. C, Visual phenotype of co-suppressed plants and wild-type (WT).
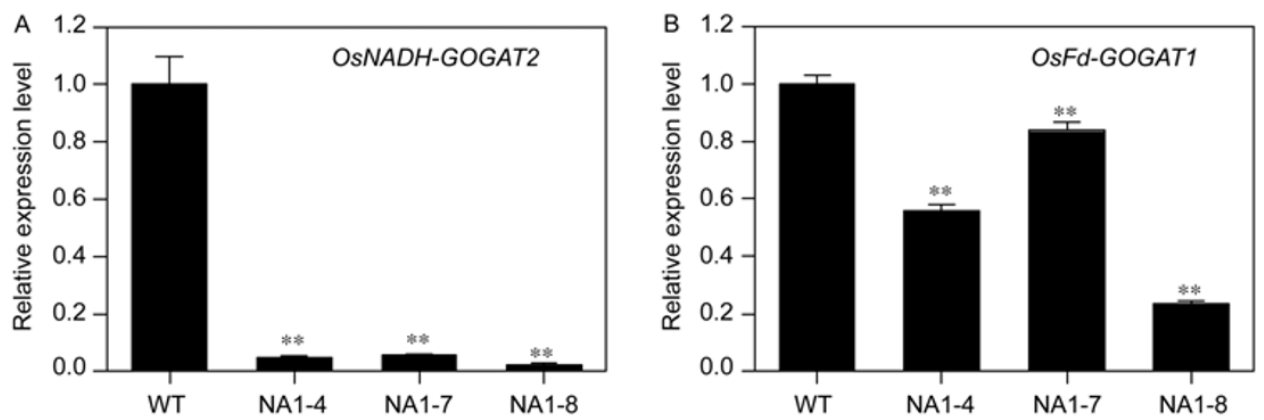

Figure 4 Relative transcription level of $O S N A D H$-GOGAT2 and $O S F d$-GOGAT1 in co-suppressed plants and the wild-type. A, Relative expression levels of $O S N A D H-G O G A T 2$ in leaves. B, Relative expression levels of $O s F d-G O G A T 1$ in leaves. Data are means $\pm \mathrm{SE}$ of three technical replicates. Two asterisks indicate significant difference from the wild-type (WT) at the level of $P<0.01$ ( $t$-test).

shoot dry weight, root dry weight, and root number compared with the wild-type in hydroponic conditions (Figure 5). Phenotypic changes were more obvious in the field; co-suppressed plants were smaller, thinner, and somewhat yellowish compared with the wild-type. Agronomic traits such as tiller number, plant height, total shoot dry weight, yield per plant, and the thousand kernel weight were all significantly decreased (Table 2, Figure 3C). Co-segregation of the transgenic fragment with phenotypic changes was confirmed by PCR analysis with specific primers (Table 1).

NADH-GOGAT activity was assayed in co-suppressed and wild-type plants. The NADH-GOGAT activity was significantly decreased in co-suppressed plants compared with that in the wild-type (Table 3 ); the reduction in leaves was $34.8 \%-70.8 \%$ and in roots was $65.8 \%-87.0 \%$.

\subsection{Changes in activities of several enzymes related to} carbon and nitrogen metabolism in co-suppressed plants

We examined the catalytic activities of some other important enzymes in carbon and nitrogen metabolism. In cosuppressed plants, only NR, leaf Fd-GOGAT, root G6PDH, and root ShikDH showed consistent changes compared with their respective activities in the wild-type. Compared with that in the wild-type, NR activity in co-suppressed plants was increased $10.5 \%-64.2 \%$ in leaves but decreased $15.6 \%-74.2 \%$ in roots. The leaf Fd-GOGAT activity was decreased $27.3 \%-54.7 \%$ compared with that in wild-type, 

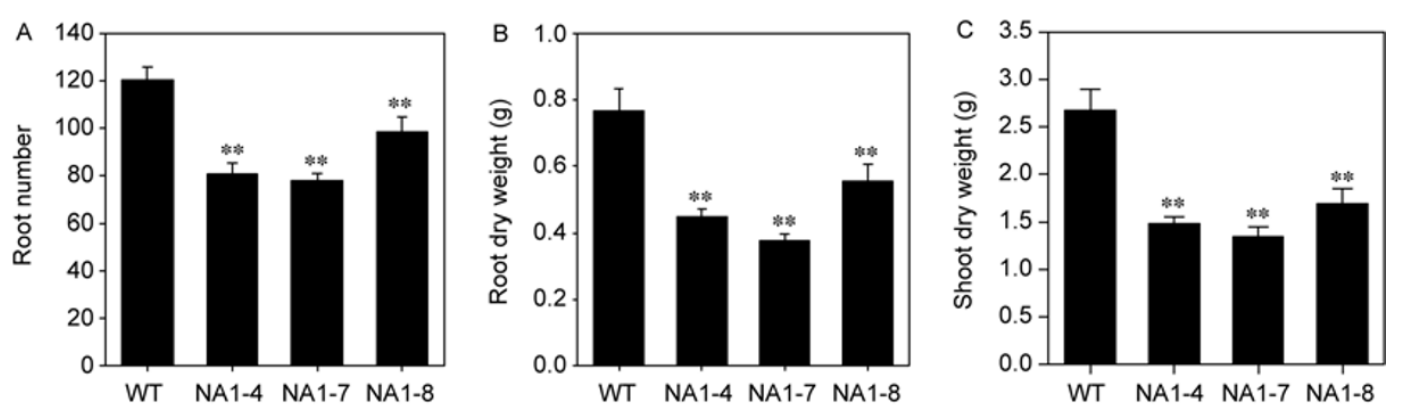

Figure 5 Morphological characteristics of GOGAT co-suppressed plants in hydroponic conditions. Plants were harvested $30 \mathrm{~d}$ after transplanting into Yoshida solution. Panels (A-C) represent root number (A), total root dry weight (B), and total shoot dry weight (C). Data are means \pm SE of at least seven individual plants per line. Two asterisks indicate values significantly different from the wild-type (WT) at the level of $P<0.01(t$-test).

Table 2 Morphological characteristics of field-grown GOGAT co-suppressed plants ${ }^{\mathrm{a}}$

\begin{tabular}{ccrrr}
\hline Trait & WT & NA1-4 & NA1-7 & NA1-8 \\
\hline Tiller number per plant & $10.04 \pm 0.34$ & $2.44 \pm 0.13^{* * *}$ & $2.52 \pm 0.15^{* *}$ & $5.00 \pm 0.30^{* *}$ \\
Plant height (cm) & $99.10 \pm 0.70$ & $79.97 \pm 0.77^{* *}$ & $81.86 \pm 0.73^{* *}$ & $79.38 \pm 3.04^{* *}$ \\
Total shoot dry weight (g) & $48.48 \pm 1.89$ & $7.01 \pm 0.47^{* *}$ & $7.40 \pm 0.42^{* *}$ & $14.32 \pm 0.95^{* *}$ \\
Yield per plant (g) & $27.77 \pm 1.14$ & $4.17 \pm 0.24^{* *}$ & $4.56 \pm 0.29^{* *}$ & $7.63 \pm 0.49^{* *}$ \\
Thousand kernel weight (g) & $2.46 \pm 0.03$ & $2.32 \pm 0.03^{* *}$ & $2.35 \pm 0.04$ & $2.28 \pm 0.03^{* *}$ \\
\hline
\end{tabular}

a) Field-grown plants were harvested after maturity. Data are means \pm SE of measurements from at least 10 independent plants per line. Two asterisks indicate values significantly different from the wild-type (WT) at the level of $P<0.01(t$-test).

Table 3 Enzyme activities in co-suppressed lines and wild-type ${ }^{\text {a) }}$

\begin{tabular}{ccccccccc}
\hline Enzyme & WT & NA1-4 & NA1-7 & NA1-8 & WT & NA1-4 & NA1-7 & NA1-8 \\
\hline & \multicolumn{7}{c}{ Leaves } \\
NADH-GOGAT & $157.7 \pm 17.7$ & $32.9 \pm 3.2^{* *}$ & $23.9 \pm 0.4^{* *}$ & $53.9 \pm 21.6^{* *}$ & $121.7 \pm 6.3$ & $58.2 \pm 8.8^{* * *}$ & $68.6 \pm 7.9^{* * *}$ & $79.4 \pm 9.4^{* *}$ \\
Fd-GOGAT & $111.8 \pm 17.1$ & $64.9 \pm 18.0^{* *}$ & $81.3 \pm 14.3$ & $50.7 \pm 15.2^{*}$ & - & - & - & - \\
NADP-MDH & $291.1 \pm 67.1$ & $295.8 \pm 55.1$ & $260.9 \pm 35.8$ & $288.7 \pm 67.1$ & - & - & - & - \\
GS & $3298.0 \pm 217.7$ & $3187.9 \pm 70.2$ & $3467.5 \pm 171.3$ & $2917.0 \pm 112.9$ & $548.9 \pm 42.2$ & $607.7 \pm 114.1$ & $714.1 \pm 148.4$ & $722.5 \pm 261.2$ \\
NR & $191.0 \pm 5.8$ & $276.1 \pm 5.8^{*}$ & $313.6 \pm 11.3^{*}$ & $211.1 \pm 31.4$ & $150.3 \pm 9.0$ & $84.7 \pm 2.3^{*}$ & $41.8 \pm 6.6^{* * *}$ & $71.2 \pm 10.3^{* * *}$ \\
NiR & $261.6 \pm 26.4$ & $295.5 \pm 12.4$ & $302.0 \pm 12.6$ & $358.0 \pm 75.4$ & $366.5 \pm 8.0$ & $338.0 \pm 11.7$ & $333.3 \pm 2.6$ & $364.2 \pm 26.3$ \\
NAD-GDH & $2039.7 \pm 24.0$ & $2019.5 \pm 60.3$ & $2149.7 \pm 22.5$ & $1921.5 \pm 72.1$ & $2969.6 \pm 101.0$ & $3338.5 \pm 83.0^{*}$ & $2621.4 \pm 25.4$ & $3143.2 \pm 121.1$ \\
NADP-GDH & $193.0 \pm 5.4$ & $191.4 \pm 12.6$ & $155.5 \pm 20.6$ & $158.7 \pm 19.4$ & $459.4 \pm 18.8$ & $555.2 \pm 24.7^{*}$ & $434.6 \pm 35.5$ & $547.0 \pm 29.2$ \\
AlaAT & $2781.1 \pm 141.6$ & $2974.8 \pm 183.9$ & $2943.3 \pm 230.5$ & $2589.8 \pm 294.8$ & $1285.6 \pm 57.3$ & $1781.3 \pm 94.9^{*}$ & $1498.1 \pm 94.1$ & $1169.7 \pm 88.4$ \\
AspAT & $5578.6 \pm 188.0$ & $5586.1 \pm 121.0$ & $5219.8 \pm 163.7$ & $5244.1 \pm 263.5$ & $3106.1 \pm 106.0$ & $3301.1 \pm 182.8$ & $3011.0 \pm 106.7$ & $2791.4 \pm 41.1$ \\
PEPCase & $1503.4 \pm 155.9$ & $884.5 \pm 201.7^{*}$ & $1104.5 \pm 116.3$ & $1236.6 \pm 61.8$ & $343.8 \pm 68.4$ & $616.4 \pm 144.7^{*}$ & $315.4 \pm 35.9$ & $367.0 \pm 32.6$ \\
NADP-ICDH & $1076.6 \pm 129.0$ & $1106.3 \pm 54.6$ & $1066.3 \pm 29.3$ & $1020.6 \pm 88.4$ & $1165.1 \pm 54.7$ & $1280.1 \pm 25.2$ & $1171.7 \pm 19.4$ & $1301.2 \pm 47.1^{*}$ \\
PK & $440.7 \pm 25.1$ & $427.9 \pm 61.8$ & $489.0 \pm 12.2$ & $462.4 \pm 21.8$ & $382.1 \pm 23.4$ & $372.8 \pm 16.6$ & $371.3 \pm 10.1$ & $408.1 \pm 9.0$ \\
ShikDH & $1508 \pm 57.5$ & $1559 \pm 64.9$ & $1547 \pm 48.0$ & $1419 \pm 78.3$ & $538.4 \pm 27.9$ & $429.9 \pm 6.1^{*}$ & $389.5 \pm 11.2^{*}$ & $517.7 \pm 36.7$ \\
G6PDH & $2768.7 \pm 199.9$ & $2475.1 \pm 89.9$ & $2408.4 \pm 28.4$ & $2597.5 \pm 78.6$ & $2267.9 \pm 86.7$ & $2027.3 \pm 124.3$ & $1747.0 \pm 188.9^{*}$ & $1841.8 \pm 82.4^{*}$ \\
FruK & $767.4 \pm 39.3$ & $771.8 \pm 33.8$ & $694.7 \pm 43.8$ & $695.7 \pm 18.5$ & $509.2 \pm 21.9$ & $563.1 \pm 27.4$ & $500.4 \pm 23.1$ & $477.4 \pm 15.0$ \\
GlcK & $462.4 \pm 21.8$ & $375.8 \pm 21.0^{*}$ & $358.7 \pm 39.7^{*}$ & $384.6 \pm 11.2$ & $719.9 \pm 34.1$ & $749.3 \pm 55.7$ & $677.1 \pm 32.8$ & $640.5 \pm 13.0$ \\
FBPase & $2707.5 \pm 174.8$ & $2629.4 \pm 12.9$ & $2691.8 \pm 103.8$ & $2584.7 \pm 132.1$ & $909.6 \pm 33.9$ & $937.8 \pm 51.4$ & $929.8 \pm 15.1$ & $842.7 \pm 26.2$ \\
\hline
\end{tabular}

a) Enzyme activities were determined in fully expanded leaves and roots sampled during the day time from hydroponic plants at the 5-6 leaf stage. Data are means $\pm \mathrm{SE}$ of measurements from three independent plants per line, with each plant tested at least three times. One and two asterisks indicate values significantly different from the wild-type (WT) at the levels of $P<0.05$ and $P<0.01$, respectively ( $t$-test). Enzyme activity values are given as nmol $\mathrm{g} \mathrm{FW}^{-1}$ $\min ^{-1} .-$, Not determined. 
which was consistent with its transcription level. There were no apparent changes in the activities of other enzymes tested (Table 3).

\subsection{Metabolite profiles were changed in co-suppressed plants}

To evaluate the effects of GOGAT suppression on nitrogen assimilation and utilization, we measured the contents of some nitrogenous compounds (Figure 6A-D). Compared with their respective contents in the wild-type, total shoot nitrogen content was decreased by $20.9 \%-23.3 \%$ in co-suppressed plants, whereas the total nitrogen content in seeds was stable. Leaf nitrate content in co-suppressed plants was also significantly decreased by $19.8 \%-26.3 \%$. Leaf soluble protein content was also decreased but not so significantly. Leaf free ammonium content was significantly increased in co-suppressed plants.

Leaf free amino acids were also determined (Table 4). Some amino acids such as Glu, Asp, Gly, and Ala were decreased significantly in co-suppressed plants, compared with their respective levels in the wild-type.

Because carbon and nitrogen metabolism are tightly coordinated in plants, we also evaluated some carbon metabolites. Compared with their respective levels in the wild-type, leaf glucose, fructose, and total soluble sugars were significantly decreased in most of the co-suppressed lines (Figure 7A-D). Co-suppressed lines also showed a slight increase in starch content (Figure 7E). Sugar phosphates were also significantly decreased (Figure 7F). Glucose-6-phosphate, fructose-6-phosphate, and glucose-1-phosphate contents were decreased by $87.4 \%-91.1 \%, 75.8 \%-91.6 \%$, and $50.7 \%-$
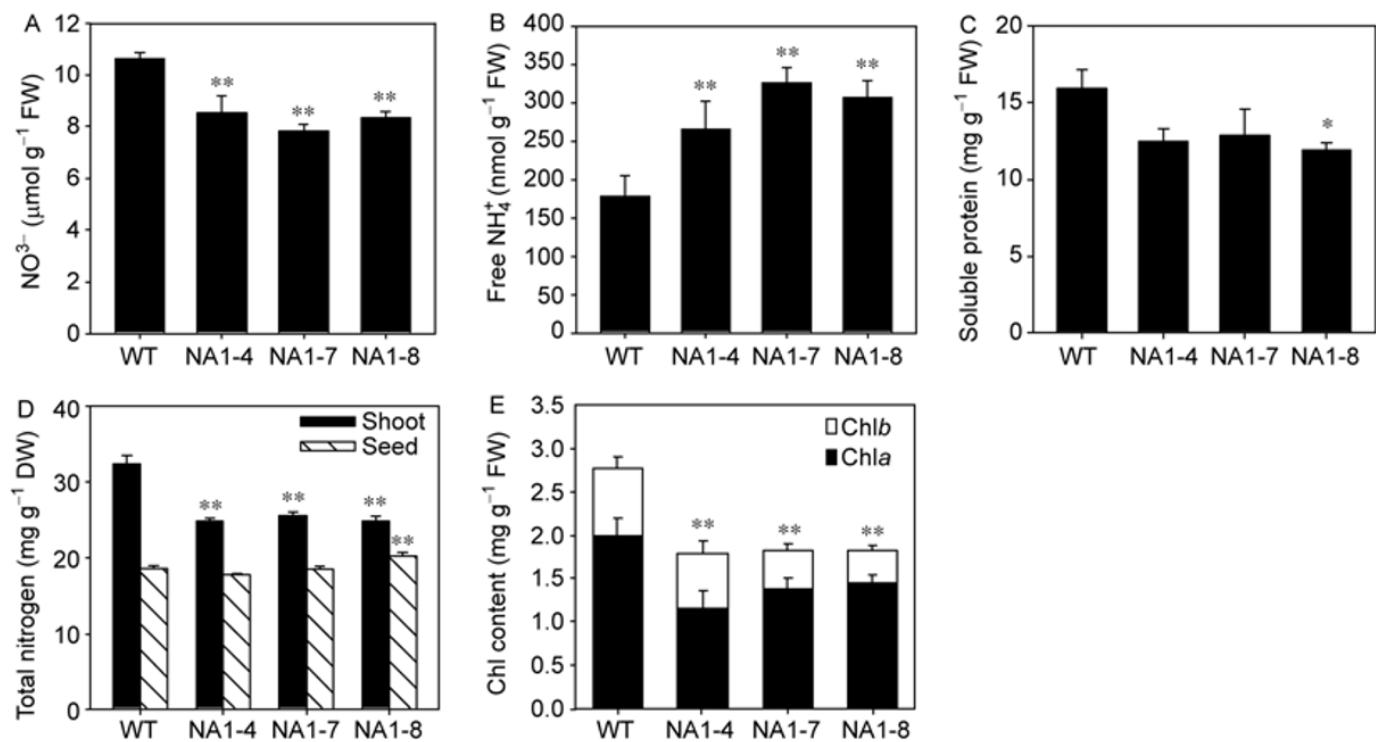

Figure 6 Nitrogenous compounds contents in co-suppressed lines and wild-type. Nitrate (A), free ammonium (B), soluble protein (C), and chlorophyll (E) in flag leaves. Total shoot and seed nitrogen (D) in field-grown plants. Data are means \pm SE of measurements from six individual plants per line. One and two asterisks indicate values significantly different from the wild-type (WT) at the levels of $P<0.05$ and $P<0.01$, respectively $(t$-test).

Table 4 Free amino acid content in leaves of co-suppressed lines and the wild-type ${ }^{\text {a) }}$

\begin{tabular}{|c|c|c|c|c|}
\hline Amino acids $\left(\mathrm{nmol} \mathrm{g}^{-1} \mathrm{FW}\right)$ & WT & NA1-4 & NA1-7 & NA1-8 \\
\hline Asp & $162.9 \pm 47.9$ & $35.7 \pm 1.6^{*}$ & $10.6 \pm 3.8^{*}$ & $36.6 \pm 8.4^{*}$ \\
\hline Thr & $11.9 \pm 6.7$ & $20.1 \pm 0.7$ & $16.0 \pm 1.5$ & $13.2 \pm 1.3$ \\
\hline Glu & $1946.7 \pm 273.0$ & $715.2 \pm 74.5^{*}$ & $511.0 \pm 23.9^{*}$ & $749.3 \pm 185.2^{*}$ \\
\hline Gly & $270.9 \pm 57.1$ & $89.9 \pm 16.6^{*}$ & $27.2 \pm 3.9^{*}$ & $145.3 \pm 20.2^{*}$ \\
\hline Ala & $727.1 \pm 134.6$ & $169.5 \pm 48.0^{*}$ & $13.1 \pm 2.3^{*}$ & $337.5 \pm 67.3^{*}$ \\
\hline Cys & $88.6 \pm 14.3$ & $56.1 \pm 2.6^{*}$ & $60.8 \pm 5.2^{*}$ & $68.2 \pm 8.8$ \\
\hline Leu & $34.8 \pm 7.9$ & $32.4 \pm 4.5$ & $34.6 \pm 4.4$ & $20.7 \pm 2.8$ \\
\hline Tyr & $71.2 \pm 6.6$ & $50.4 \pm 2.1^{*}$ & $56.1 \pm 4.8$ & $59.2 \pm 4.7$ \\
\hline Phe & $40.2 \pm 5.7$ & $33.8 \pm 1.3$ & $15.0 \pm 5.5^{*}$ & $33.9 \pm 0.6$ \\
\hline Lys & $12.9 \pm 3.3$ & $11.0 \pm 3.7$ & $4.2 \pm 0.8^{*}$ & $6.8 \pm 2.5$ \\
\hline $\operatorname{Arg}$ & $6.0 \pm 5.3$ & $5.7 \pm 3.1$ & $2.4 \pm 1.2$ & $2.8 \pm 1.5$ \\
\hline
\end{tabular}

a) The contents of free amino acids were determined in flag leaves sampled from field-grown plants approximately $38 \mathrm{~d}$ after transplanting. Data are means $\pm \mathrm{SE}$ of measurements from three independent plants per line. * indicates significant difference from WT $(P<0.05, t$-test $)$. 
$73.6 \%$, respectively, in co-suppressed lines.

The substrate of NADH-GOGAT and also the carbon skeleton for Glu and Gln synthesis is 2-OG. The 2-OG content in leaves was significantly increased in most co-suppressed lines compared with that in wild-type; in the leaf, the 2-OG precursor, isocitrate, showed the same trend. The contents of OAA, malate, and pyruvate were also increased in some, but not all, co-suppressed lines (Figure 8).

Pyridine nucleotides are not only nitrogenous compounds, but also redox-related compounds. Co-suppressed plants showed lower levels of four kinds of leaf pyridine nucleo- tides compared with the wild-type. The levels of $\mathrm{NADP}^{+}$ and $\mathrm{NAD}^{+}$, the major components of pyridine nucleotides, were decreased to a greater extent than those of NADPH and $\mathrm{NADH}$. Thus, the ratios of $\mathrm{NADP}^{+} / \mathrm{NADPH}$ and $\mathrm{NAD}^{+} /$ $\mathrm{NADH}$ were also decreased, especially at night (Figure 9A-F).

\subsection{Chlorophyll content and $F_{v} / F_{m}$ were decreased in co-suppressed lines}

Compared with their respective contents in the wild-type,
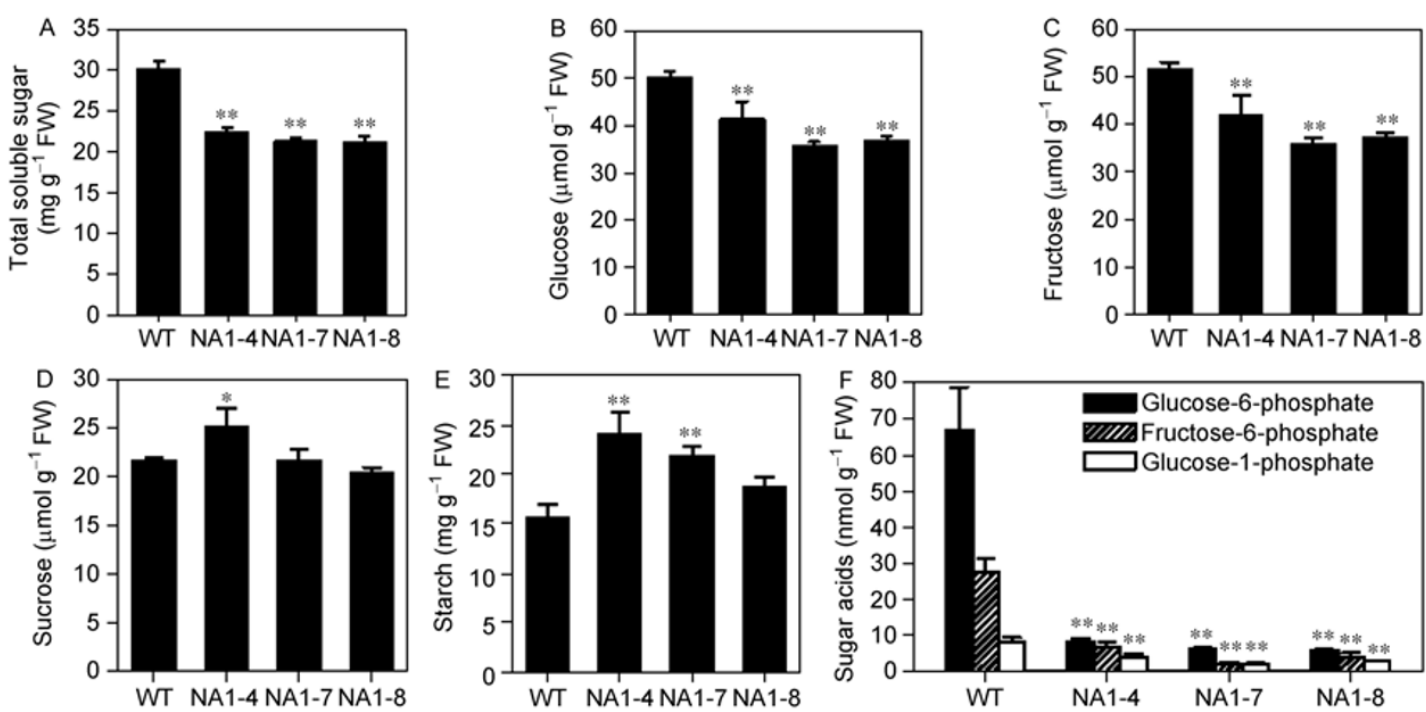

Figure 7 Sugars and sugar phosphates contents in co-suppressed lines and wild-type. The contents of total soluble sugar (A), glucose (B), fructose (C), sucrose (D), starch (E), and sugar phosphates (F) were determined in flag leaves of field-grown plants. Data are means \pm SE of measurements from at least three individual plants per line. One and two asterisks indicate values significantly different from the wild-type (WT) at the levels of $P<0.05$ and $P<0.01$, respectively $(t$-test).
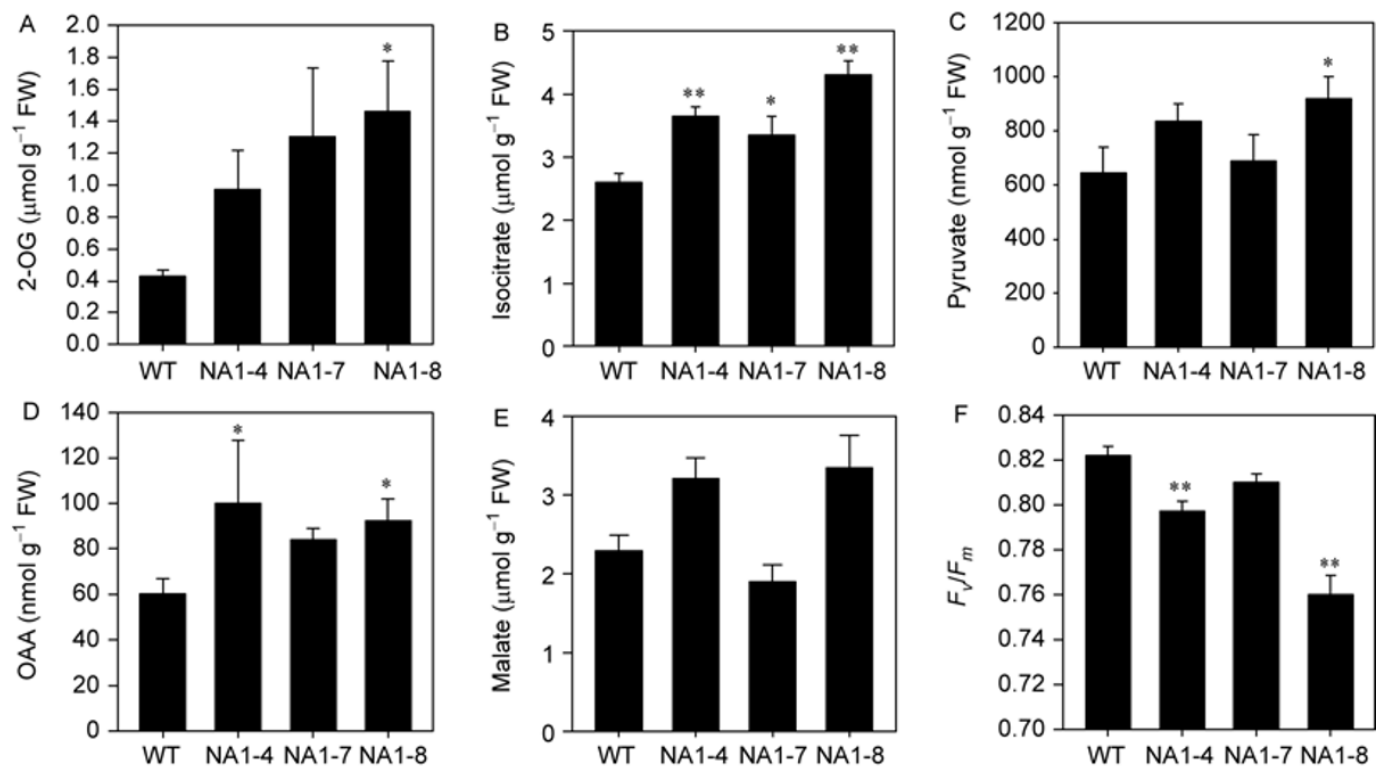

Figure 8 Organic acids contents and $F_{v} / F_{m}$ of co-suppressed lines and the wild-type. 2-OG (A), isocitrate (B), pyruvate (C), OAA (D), and malate (E) contents were determined in flag leaves of field-grown plants. Flag leaf $F_{v} / F_{m}(\mathrm{~F})$ was also determined at this stage. Data are means \pm SE of measurements from at least three individual plants per line. One and two asterisks indicate values significantly different from the wild-type (WT) at the levels of $P<0.05$ and $P<0.01$, respectively $(t$-test $)$. 

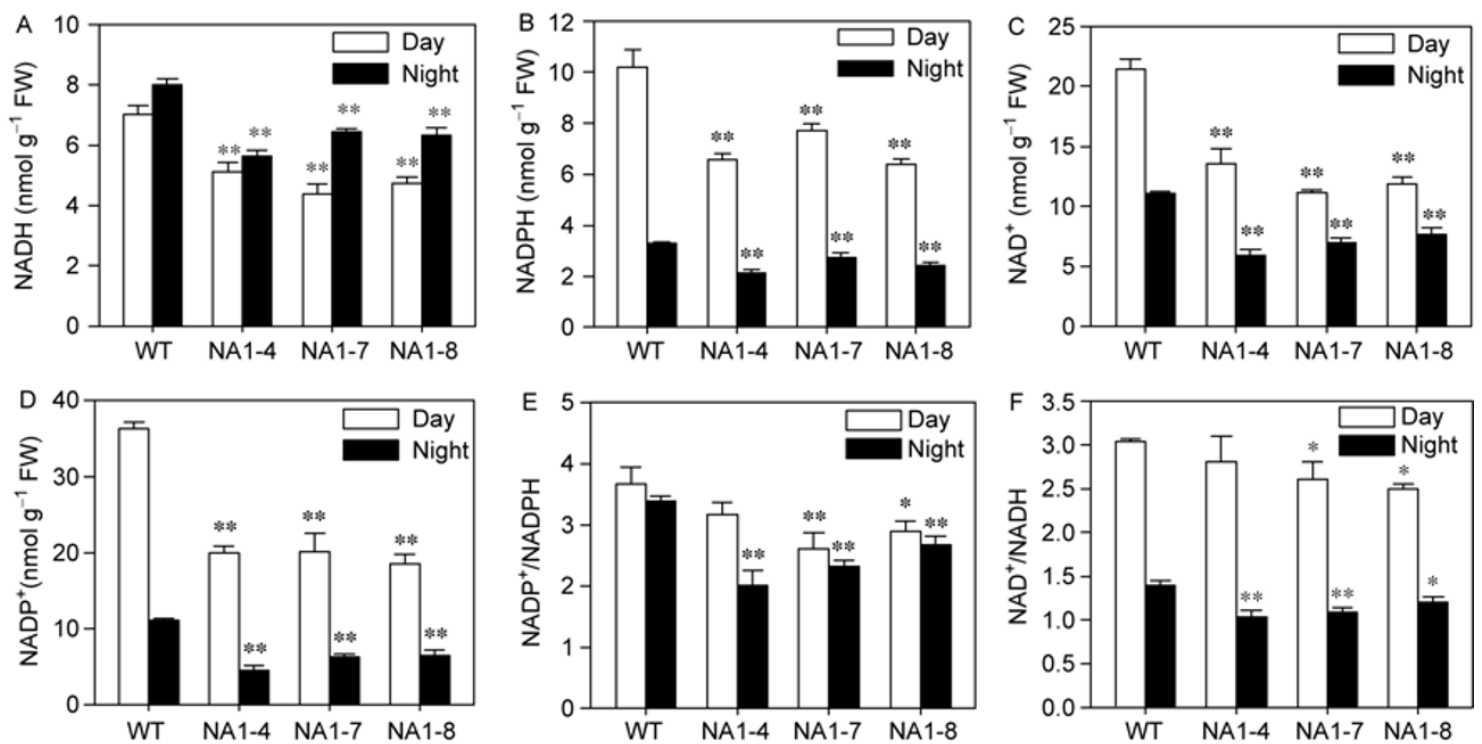

Figure 9 Pyridine nucleotide levels in co-suppressed lines and wild-type. NADH (A), NADPH (B), NAD ${ }^{+}(\mathrm{C}), \mathrm{NADP}^{+}(\mathrm{D}), \mathrm{NADP}^{+} / \mathrm{NADPH}(\mathrm{E})$, and $^{-}$ $\mathrm{NAD}^{+} / \mathrm{NADH}(\mathrm{F})$ were determined in flag leaves of field-grown plants sampled in the daytime or at night. Data are means \pm SE of measurements from six individual plants per line. One and two asterisks indicate values significantly different from the wild-type (WT) at levels of $P<0.05$ and $P<0.01$, respectively ( $t$-test).

chl $a$, chl $b$, and total chlorophyll contents were significantly decreased in co-suppressed plants (Figure 6E), which was consistent with their yellowish leaf phenotype. The ratio of $F_{v} / F_{m}$ was much lower in leaves of co-suppressed plants (Figure $8 \mathrm{~F}$ ) than in the wild-type, indicating that co-suppressed plants may have decreased ability to perform photosynthesis.

\section{Discussion}

\subsection{Phenotypic changes resulting from suppression of NADH-GOGATS}

In our research, less than $1 / 3$ of the $T_{0}$ transgenic plants exhibited severely changed phenotypes compared with the wild-type. Our results suggest that these phenotypic changes resulted from co-suppression. First, plants with altered phenotypes showed faint main bands and strong smear signals in Northern blot analyses (Figure 3A), indicating that the transcripts of the transgene were at least partially degraded in these plants. Second, although we could not evaluate the endogenous OsNADH-GOGAT1 transcripts because they were indistinguishable from the transgene, we evaluated other gene members of this family: $O s$ $N A D H-G O G A T 2$ and OsFd-GOGAT1, which share high sequence identity with OSNADH-GOGAT1, were significantly suppressed in plants with phenotypic changes. The high similarity in the coding sequence satisfied the requirement of $60 \%-70 \%$ identity to achieve posttranscriptional gene silencing, as suggested by Kunz et al. [40]. The transgene and endogenous OSNADH-GOGATI have the same coding sequence, and the suppression of OSNADH-GOGAT2 and OsFd-GOGATI suggested that the endogenous Os$N A D H-G O G A T 1$ was also suppressed in these plants. Third, OsNADH-GOGAT1 was mainly expressed in roots and OsNADH-GOGAT2 was mainly expressed in the leaf and leaf sheath. Accordingly, the enzyme activities of the NADH-GOGAT enzyme were significantly decreased in roots and leaves of phenotype-altered plants (Table 3), indicating that OsNADH-GOGAT1 and OsNADH-GOGAT2 were simultaneously suppressed in these plants. Fourth, sense expression of an OSNADH-GOGAT2 fragment in rice under the control of the CaMV35S promoter also caused co-suppression, and co-suppressed plants showed the same phenotypic changes and reductions in enzyme activities as those found in this research. The low expression level of GOGATs in co-suppressed plants was identified by real-time RT-PCR (data not shown).

It is possible that $O S N A D H-G O G A T s$ are tightly monitored and regulated in vivo and their threshold for gene silencing was very low. In that case, the mRNA level determined by our northern blot analyses would have been out of the range for gene silencing. Yamaya et al. [19] also obtained OSNADH-GOGAT1 co-suppressed indica rice using a japonica NADH-GOGAT1 cDNA driven by its own promoter. Only low levels of NADH-GOGAT mRNA accumulated in transgenic tobacco overexpressing alfalfa $N A D H$ GOGAT cDNA, which was detected by RT-PCR analysis at the transcription level rather than by northern blot [18]. Co-suppressions have also been reported for other nitrogen assimilation-related genes such as $N R, N i R$, and $G S 2$ [41,42].

$N A D H-G O G A T$ is thought to be responsible for primary nitrogen assimilation in roots, and it is also the most important step in the nitrogen assimilation process [2]. Suppres- 
sion of this gene decreased the nitrogen assimilation rate in rice and caused nitrogen-starvation-like phenotypes, for example, fewer roots, and low shoot and root dry weight, even in nitrogen-sufficient hydroponic conditions (Figure 5). Limited nitrogen assimilation also affected agronomic characteristics of field-grown plants including yield per plant and thousand kernel weight (Table 2). Other studies have also reported that OsNADH-GOGAT1 affects tiller number and yield per plant [19]. A similar phenotype was reported in glt1-T mutant plants [6]. Suppression of OsFd-GOGAT1 may be another reason for the phenotypic change, because of the decreased ability to re-assimilate ammonium liberated by photorespiration.

\subsection{NADH-GOGAT and nitrogen metabolism}

NADH-GOGAT can affect nitrogen metabolism directly through Glu and Gln. We assume that absorbed nitrate was reduced to ammonium through NR and NiR. These ammonium compounds combine with ammonium directly absorbed from soil, and are incorporated into Glu through the GS/NADH-GOGAT cycle [1]. Glu is the original material from which other amino acids and organic nitrogenous compounds are formed [2]. In our research, suppression of the NADH-GOGAT genes significantly decreased the free Glu content in the leaves (Table 4). Other kinds of amino acids and nitrogenous compounds such as chlorophyll and pyridine nucleotides were also decreased significantly (Table 4, Figures 6 and 9), and consequently, total shoot nitrogen content was also decreased (Figure 6D). Decreases in the Glu and total nitrogen contents were also reported in transgenic alfalfa plants with decreased NADH-GOGAT activity and in Arabidopsis glt1-T mutant plants [6,43].

The observed effects may also be because of changes in the activities of nitrogen assimilation-related enzymes. In the case of inorganic nitrogen compounds, nitrate was decreased but ammonium increased in leaves of co-suppressed plants (Figure 6A and B). These changes may be because of increased NR activity in leaves (Table 3), which accelerated the formation of ammonium from nitrate. Ammonium accumulation may also be partly attributed to the decreased Fd-GOGAT activity in leaves (Table 3), which decreased the efficiency of re-assimilation of ammonium liberated from photorespiration. The activities of Fd-GOGAT and NR were closely correlated with the content of some nitrogenous compounds, but most of the other nitrogen-related enzymes were not altered in co-suppressed plants (Table 3, Figure 6). The stability of these enzymes may benefit plants during adaption to low nitrogen conditions.

In rice, GS1;2 activity couples with that of NADHGOGAT1 to achieve primary nitrogen assimilation in roots [11]. In our research, however, GS activity in roots was unchanged (Table 3). Another suggested function of rice NADH-GOGAT1 is to work with GS1;1 to reuse remobilized nitrogen for grain development [11], but we found that total nitrogen content was not significantly altered in grains of co-suppressed plants (Figure 6D). It is possible that the residual enzyme activity provided enough nitrogen for seed development, or that seeds obtained nitrogen from other metabolic pathways.

\subsection{NADH-GOGAT and carbon metabolism}

GOGAT can affect carbon metabolism through 2-OG. As a kind of carbon skeleton, 2-OG is incorporated into Glu through a reaction catalyzed by GOGAT. 2-OG is also an intermediate metabolite in the TCA cycle and is synthesized by mitochondrial-located NAD-IDH or the cytosol-located NADP-ICDH [44]. In our research, suppression of GOGAT affected carbon metabolism, resulting in increases in leaf 2-OG and isocitrate (Figure $8 \mathrm{~A}$ and $\mathrm{B}$ ); the former is the substrate of GOGAT, and the latter is the substrate of NAD-IDH and NADP-ICDH. NAD-IDH and NADP-ICDH catalyze the formation of 2-OG from isocitrate using $\mathrm{NAD}^{+}$ or $\mathrm{NADP}^{+}$as cofactors, respectively. The accumulation of 2-OG showed that the reactions catalyzed by GOGAT were the main route of 2-OG consumption. 2-OG can also shuttle among subcellular compartments through translocators [45], and this accumulation will affect other 2-OG-related processes such as the TCA cycle. The slight alterations in levels of other kinds of organic acids in leaves may be consequences of such effects (Figure 8C-E).

The contents of leaf soluble sugars and sugar phosphates were significantly decreased in co-suppressed lines (Figure 7). This was probably because of the decreased ability to perform photosynthesis because of the shortage of leaf chlorophyll (Figure 6E). These results also demonstrate that carbon and nitrogen metabolisms are tightly coordinated in rice.

\subsection{NADH-GOGAT and pyridine nucleotides status}

In our research, four kinds of pyridine nucleotides were significantly decreased in co-suppressed plants; the ratios of $\mathrm{NAD}^{+} / \mathrm{NADH}$ and $\mathrm{NADP}^{+} / \mathrm{NADPH}$ were also decreased in most lines compared with wild-type plants (Figure 9). These results indicated that co-suppressed plants could not synthesize enough organic nitrogenous compounds, including pyridine nucleotides. The decreased ratio of $\mathrm{NAD}^{+} / \mathrm{NADH}$ can be partially explained by the decreased activity of NADH-GOGAT, which uses NADH as a cofactor. NADPH can be produced from NADH by NADH kinase, which is located in the chloroplast, cytosol, and mitochondria [46]. Changes in $\mathrm{NADP}^{+} / \mathrm{NADPH}$ may be attributed to NADH kinase or dehydrogenases such as NAD-IDH and NADP$\mathrm{ICDH}$, which share the same substrates but have different cofactors.

Pyridine nucleotides such as $\mathrm{NAD}(\mathrm{H})$ and $\mathrm{NADP}(\mathrm{H})$ are not only nitrogenous compounds but also serve as cofactors in numerous energy-producing and biosynthetic reactions. 
They play crucial roles in redox signaling linked to stress and development [46]. Other carbon and nitrogen metabolism-related enzymes such as NR, NiR, GDH, and AspAT also need redox equivalents to be supplied. It was reported in the tobacco mutant CMS that modification of pyridine nucleotide status affected nitrate assimilation and the integration of carbon and nitrogen metabolism [34]. Metabolic profiles also changed in transgenic potato differentially expressing the external mitochondrial NADPH dehydrogenase [47]. NADHGOGAT1 is predicted to be present in the plastids [3]; the altered content of $\mathrm{NAD}(\mathrm{H})$ affects the TCA cycle enzymes through translocation of substrates such as 2-OG and citrate between the cytosol and organelles [45,48]. As reported previously, repression of mitochondrial enzymes results in changes to metabolic profiles [49]. In this research, the observed changes of carbon and nitrogen metabolism in co-suppressed plants may be partly attributed to the modified pyridine nucleotide status.

This work was supported by the National High Technology Research and Development Program of China (Grant No. 2010AA101802) and Transgenic Project (Grant No. 2008ZX08001-005).

1 Andrews M, Lea P J, Raven J A, et al. Can genetic manipulation of plant nitrogen assimilation enzymes result in increased crop yield and greater N-use efficiency? An assessment. Ann Appl Biol, 2004, 145: $25-40$

2 Lam H M, Coschigano K T, Oliveira I C, et al. The molecular-genetics of nitrogen assimilation into amino acids in higher plants. Annu Rev Plant Physiol Plant Mol Biol, 1996, 47: 569-593

3 Goto S, Akagawa T, Kojima S, et al. Organization and structure of NADH-dependent glutamate synthase gene from rice plants. Biochim Biophys Acta, 1998, 1387: 298-308

4 Blanco L, Reddy P M, Silvente S, et al. Molecular cloning, characterization and regulation of two different NADH-glutamate synthase cDNAs in bean nodules. Plant Cell Environ, 2008, 31: 454-472

5 Coschigano K T, Melo-Oliveira R, Lim J, et al. Arabidopsis gls mutants and distinct Fd-GOGAT genes: implications for photorespiration and primary nitrogen assimilation. Plant Cell, 1998, 10: 741-752

6 Lancien M, Martin M, Hsieh M H, et al. Arabidopsis glt1-T mutant defines a role for NADH-GOGAT in the non-photorespiratory ammonium assimilatory pathway. Plant J, 2002, 29: 347-358

7 Suzuki A, Vidal J, Gadal P. Glutamate synthase isoforms in rice: immunological studies of enzymes in green leaf, etiolated leaf, and root tissues. Plant Physiol, 1982, 70: 827-832

8 Ishiyama K, Hayakawa T, Yamaya T. Expression of NADH-dependent glutamate synthase protein in the epidermis and exodermis of rice roots in response to the supply of ammonium ions. Planta, 1998, 204: 288-294

9 Ishiyama K, Kojima S, Takahashi H, et al. Cell type distinct accumulations of mRNA and protein for NADH-dependent glutamate synthase in rice roots in response to the supply of $\mathrm{NH}_{4}^{+}$. Plant Physiol Biochem, 2003, 41: 643-647

10 Abiko T, Obara M, Ushioda A, et al. Localization of NAD-isocitrate dehydrogenase and glutamate dehydrogenase in rice roots: candidates for providing carbon skeletons to NADH-glutamate synthase. Plant Cell Physiol, 2005, 46: 1724-1734

11 Tabuchi M, Abiko T, Yamaya T. Assimilation of ammonium ions and reutilization of nitrogen in rice (Oryza sativa L.). J Exp Bot, 2007, 58: 2319-2327

12 Yamaya T, Hayakawa T, Tanasawa K, et al. Tissue distribution of glutamate synthase and glutamine synthetase in rice leaves: occur- rence of NADH-dependent glutamate synthase protein and activity in the unexpanded, nongreen leaf blades. Plant Physiol, 1992, 100: 1427-1432

13 Hayakawa T, Yamaya T, Mae T, et al. Changes in the content of two glutamate synthase proteins in spikelets of rice (Oryza sativa) plants during ripening. Plant Physiol, 1993, 101: 1257-1262

14 Hayakawa T, Nakamura T, Hattori F, et al. Cellular localization of NADH-dependent glutamate-synthase protein in vascular bundles of unexpanded leaf blades and young grains of rice plants. Planta, 1994, 193: 455-460

15 Yamaya T, Tanno H, Hirose N, et al. A supply of nitrogen causes increase in the level of NADH-dependent glutamate synthase protein and in the activity of the enzyme in roots of rice seedlings. Plant Cell Physiol, 1995, 36: 1197-1204

16 Hayakawa T, Hopkins L, Peat L J, et al. Quantitative intercellular localization of NADH-dependent glutamate synthase protein in different types of root cells in rice plants. Plant Physiol, 1999, 119: 409-416

17 Good A G, Shrawat A K, Muench D G. Can less yield more? Is reducing nutrient input into the environment compatible with maintaining crop production? Trends Plant Sci, 2004, 9: 597-605

18 Chichkova S, Arellano J, Vance C P, et al. Transgenic tobacco plants that overexpress alfalfa NADH-glutamate synthase have higher carbon and nitrogen content. J Exp Bot, 2001, 52: 2079-2087

19 Yamaya T, Obara M, Nakajima H, et al. Genetic manipulation and quantitative-trait loci mapping for nitrogen recycling in rice. J Exp Bot, 2002, 53: 917-925

20 Hiei Y, Ohta S, Komari T, et al. Efficient transformation of rice (Oryza sativa L.) mediated by Agrobacterium and sequence analysis of the boundaries of the T-DNA. Plant J, 1994, 6: 271-282

21 Yoshida S, Forno D, Cock J. Laboratory manual for physiological studies of rice. Manila: Gomez International Rice Research Institute, 1976. 61-65

22 Sambrook J, Russell D W. Molecular Cloning: A Laboratory Manual. 3rd ed. New York: Cold Spring Harbor Laboratory, 2001. 516-588

23 Hecht U, Oelmüller R, Schmidt S, et al. Action of light, nitrate and ammonium on the levels of NADH- and ferredoxin-dependent glutamate synthases in the cotyledons of mustard seedlings. Planta, 1988, 175: $130-138$

24 Gibon Y, Blaesing O E, Hannemann J, et al. A robot-based platform to measure multiple enzyme activities in Arabidopsis using a set of cycling assays: comparison of changes of enzyme activities and transcript levels during diurnal cycles and in prolonged darkness. Plant Cell, 2004, 16: 3304-3325

25 Migge A, Carrayol E, Kunz C, et al. The expression of the tobacco genes encoding plastidic glutamine synthetase or ferredoxin-dependent glutamate synthase does not depend on the rate of nitrate reduction, and is unaffected by suppression of photorespiration. J Exp Bot, 1997, 48: 1175-1181

26 Scheible W R, Gonzalez-Fontes A, Lauerer M, et al. Nitrate acts as a signal to induce organic acid metabolism and repress starch metabolism in tobacco. Plant Cell, 1997, 9: 783-798

27 Turano F J, Dashner R, Upadhyaya A, et al. Purification of mitochondrial glutamate dehydrogenase from dark-grown soybean seedlings. Plant Physiol, 1996, 112: 1357-1364

28 Jenner H L, Winning B M, Millar A H, et al. NAD malic enzyme and the control of carbohydrate metabolism in potato tubers. Plant Physiol, 2001, 126: 1139-1149

29 Borsani J, Budde C O, Porrini L, et al. Carbon metabolism of peach fruit after harvest: changes in enzymes involved in organic acid and sugar level modifications. J Exp Bot, 2009, 60: 1823-1837

30 Schneidereit J, Häusler R E, Fiene G, et al. Antisense repression reveals a crucial role of the plastidic 2-oxoglutarate/malate translocator DiT1 at the interface between carbon and nitrogen metabolism. Plant J, 2006, 45: 206-224

31 Gibon Y, Vigeolas H, Tiessen A, et al. Sensitive and high throughput metabolite assays for inorganic pyrophosphate, ADPGlc, nucleotide phosphates, and glycolytic intermediates based on a novel enzymic cycling system. Plant J, 2002, 30: 221-235

32 Novitskaya L, Trevanion S J, Driscoll S, et al. How does photorespi- 
ration modulate leaf amino acid contents? A dual approach through modelling and metabolite analysis. Plant Cell Environ, 2002, 25: 821-835

33 Gibon Y, Larher F. Cycling assay for nicotinamide adenine dinucleotides: $\mathrm{NaCl}$ precipitation and ethanol solubilization of the reduced tetrazolium. Anal Biochem, 1997, 251: 153-157

34 Dutilleul C, Lelarge C, Prioul J L, et al. Mitochondria-driven changes in leaf NAD status exert a crucial influence on the control of nitrate assimilation and the integration of carbon and nitrogen metabolism. Plant Physiol, 2005, 139: 64-78

35 Wang F, Sanz A, Brenner M L, et al. Sucrose synthase, starch accumulation, and tomato fruit sink strength. Plant Physiol, 1993, 101: 321-327

36 Geigenberger P, Reimholz R, Geiger M, et al. Regulation of sucrose and starch metabolism in potato tubers in response to short-term water deficit. Planta, 1997, 201: 502-518

37 Bradford M M. A rapid and sensitive method for the quantitation of microgram quantities of protein utilizing the principle of protein binding. Anal Biochem, 1976, 72: 248-254

38 Livak K J, Schmittgen T D. Analysis of relative gene expression data using real-time quantitative PCR and the $2^{-\Delta \Delta C_{\mathrm{T}}}$ method. Methods, 2001, 25: 402-408

39 Werner T, Holst K, Pörs Y, et al. Cytokinin deficiency causes distinct changes of sink and source parameters in tobacco shoots and roots. $\mathrm{J}$ Exp Bot, 2008, 59: 2659-2672

40 Kunz C, Schöb H, Leubner-Metzger G, et al. $\beta-1,3-$-Glucanase and chitinase transgenes in hybrids show distinctive and independent patterns of posttranscriptional gene silencing. Planta, 2001, 212: 243-249
41 Oliveira I C, Brears T, Knight T J, et al. Overexpression of cytosolic glutamine synthetase. Relation to nitrogen, light, and photorespiration. Plant Physiol, 2002, 129: 1170-1180

42 Palauqui J C, Vaucheret H. Transgenes are dispensable for the RNA degradation step of cosuppression. Proc Natl Acad Sci USA, 1998, 95: 9675-9680

43 Schoenbeck M A, Temple S J, Trepp G B, et al. Decreased NADH glutamate synthase activity in nodules and flowers of alfalfa (Medicago sativa L.) transformed with an antisense glutamate synthase transgene. J Exp Bot, 2000, 51: 29-39

44 Gálvez S, Roche O, Bismuth E, et al. Mitochondrial localization of a NADP-dependent isocitrate dehydrogenase isoenzyme by using the green fluorescent protein as a marker. Proc Natl Acad Sci USA, 1998, 95: 7813-7818

45 Weber A, Flügge U I. Interaction of cytosolic and plastidic nitrogen metabolism in plants. J Exp Bot, 2002, 53: 865-874

46 Noctor $\mathrm{G}$, Queval G, Gakière $\mathrm{B}$. NAD $(\mathrm{P})$ synthesis and pyridine nucleotide cycling in plants and their potential importance in stress conditions. J Exp Bot, 2006, 57: 1603-1620

47 Liu Y J, Nunes-Nesi A, Wallström S V, et al. A redox-mediated modulation of stem bolting in transgenic Nicotiana sylvestris differentially expressing the external mitochondrial NADPH dehydrogenase. Plant Physiol, 2009, 150: 1248-1259

48 Popova T N, Pinheiro de, Carvalho M A. Citrate and isocitrate in plant metabolism. Biochim Biophys Acta, 1998, 1364: 307-325

49 Nunes-Nesi A, Carrari F, Gibon Y, et al. Deficiency of mitochondrial fumarase activity in tomato plants impairs photosynthesis via an effect on stomatal function. Plant J, 2007, 50: 1093-1106

Open Access This article is distributed under the terms of the Creative Commons Attribution License which permits any use, distribution, and reproduction in any medium, provided the original author(s) and source are credited. 